\title{
Inference of Bacterial Pathogen Instantaneous Population Growth Dynamics
}

\author{
Anne Sicard, ${ }^{1}$ Andreina I. Castillo, ${ }^{1}$ Michael Voeltz, ${ }^{1}$ Hongyu Chen, ${ }^{2}$ Adam R. Zeilinger, ${ }^{1}$ \\ Leonardo De La Fuente,$^{2}$ and Rodrigo P. P. Almeida ${ }^{1,+}$ \\ ${ }^{1}$ Department of Environmental Science, Policy and Management, University of California, Berkeley, CA, U.S.A. \\ ${ }^{2}$ Department of Entomology and Plant Pathology, Auburn University, Auburn, AL, U.S.A.
}

Accepted 20 December 2019.

\begin{abstract}
Although bacterial host colonization is a dynamic process that requires population growth, studies often focus on comparing bacterial populations at a given time point. However, this may not reflect the dynamics of the colonization process. Timecourse assays provide important insights into the dynamics of host colonization but are laborious and may still lack resolution for immediate processes affecting populations. An alternative way to address this issue, using widely accessible tools (such as quantitative PCR [qPCR]), is to take advantage of the relationship between bacterial chromosomal replication and cell division to determine population growth status at the sampling time. Conceptually, the ratio between the number of copies at the origin of replication and that at the terminus of replication should be correlated with the measured bacterial growth rate. This peak-to-trough ratio (PTR) to estimate instantaneous population growth status was tested with the slow-growing plant-pathogenic bacterium Xylella fastidiosa. We found no correlation between PTR and the measured growth rate when using genome-level data but overall sequencing depth of coverage trends matched theoretical expectations. On the other hand, the population growth status of $X$. fastidiosa was predicted by PTR when using qPCR data, which was improved by the pretreatment of cells with a photoreactive DNA-binding dye. Our results suggest that PTR could be used to determine $X$. fastidiosa growth status both in planta and in insect vectors. We expect PTR will perform better with fast-growing bacterial pathogens, potentially becoming a powerful tool for easily and quickly assessing population growth status.
\end{abstract}

Keywords: genomics, population biology

${ }^{\dagger}$ Corresponding author: R. P. P. Almeida; rodrigoalmeida@berkeley.edu

Funding: This work used the Vincent J. Coates Genomics Sequencing Laboratory at the University of California-Berkeley, supported by a National Institutes of Health S10 OD018174 Instrumentation Grant. A. Sicard was supported by a Marie Skłodowska-Curie Fellowship (European Union's Horizon 2020 Research and Innovation Program, grant agreement 707013). The research was supported by an award from the California Department of Food and Agriculture Pierce's Disease Research program.

*The $\boldsymbol{e}$-Xtra logo stands for "electronic extra" and indicates that one supplementary figure and six supplementary tables are published online.

The author(s) declare no conflict of interest.

(C) 2020 The American Phytopathological Society
Bacterial population growth is regulated by biotic and abiotic factors. The ability to determine population growth rate during host infection is key to understanding how various factors affect host colonization. Although bacterial population growth has been extensively studied in vitro, renewed interest for such studies in vivo has been driven by fundamental questions such as host colonization processes (Smith 1998). However, that remains a challenging topic to investigate, particularly for slowgrowing organisms (Gallagher et al. 2018).

Bacterial cell division is preceded by the replication of its chromosome, effectively linking replication and population growth (Reyes-Lamothe and Sherratt 2019). Bacterial replication forks start from a single origin of replication from which they proceed in both strands until they reach the terminus where they meet, and the two chromosomes split from each other. As a result, chromosomal replication results in a larger number of DNA copies of the origin of replication than of the terminus if a population is growing in size (Fig. 1) (Rocha 2008). It has recently been demonstrated that the ratio between the number of DNA copies at the origin and terminus of replication (i.e., peak-to-trough ratio [PTR]) was correlated with the measured growth rate of a fast-growing bacterial population (Korem et al. 2015). That study was based on the analysis of sequencing coverage depth derived from metagenomic data, and was tested both in vitro and in vivo for several human bacterial species belonging to different phyla. Although nextgeneration sequencing is routinely used in some laboratories, quantitative PCR or real-time PCR (qPCR) remains the method of choice to estimate bacterial population size and, more generally, to quantify nucleic acid for large numbers of samples (Bustin et al. 2009). It is also routinely and widely used in plant pathology research for pathogen detection, among other uses.

Xylella fastidiosa is a plant-pathogenic bacterium with a large host range that includes 563 plant species (European Food Safety Authority (EFSA) 2018). This bacterial species is the causal agent of a number of diseases of economic importance, including Pierce's disease of grapevine (Almeida and Nunney 2015). The slow-growing nature of this bacterium makes it difficult to study both in vitro and in vivo. Indeed, $X$. fastidiosa can take 3 to 4 weeks to develop colonies in vitro, depending on the growth medium and strain (Almeida and Purcell 2003; Almeida et al. 2001; Saponari et al. 2017). The development of a tool enabling quick assessment of population growth status would help determine whether a certain environment (e.g., plant species, climatic conditions, and so on) is suitable for multiplication, as well as to test the effect of various treatments aimed at limiting $X$. fastidiosa growth. These and many other 
questions have traditionally used growth curves which, although valuable and informative, require sampling at multiple time points and are labor intensive. Furthermore, growth curves may be difficult to apply to studies considering pathogen colonization of vascular tissues, for example, due to biotic and abiotic heterogeneity that may affect bacterial population growth over short periods of time. Biosensors have been used to address related questions (Becerro et al. 2015; Gfeller et al. 2005; Narang et al. 2018) but their availability and ease of use is not widespread. Thus, new tools to assess bacterial population growth could help answer a broad range of fundamental and applied questions.

We tested whether PTR could predict changes in $X$. fastidiosa abundance in vitro and in vivo. This was assessed using two different $X$. fastidiosa subsp. fastidiosa strains (STL and Temecula1) causing Pierce's disease. We demonstrate that a qPCR-based PTR correlates with $X$. fastidiosa growth in vitro and genome depth of coverage of the $X$. fastidiosa genome follows hypothesized expectations. We also tested whether this approach provided insights into the status of bacterial populations within plant hosts and insect vectors.

\section{RESULTS}

\section{PTR estimated with genome coverage depth} was not correlated with population growth.

Our first goal was to determine whether PTR could be used to predict $X$. fastidiosa growth dynamics at the genome scale, and whether $X$. fastidiosa sequencing depth of coverage followed expectations based on observations of fast-growing bacteria. Explicitly, we assessed whether regions near the origin of replication had a higher depth of coverage than regions at the terminus of replication during population growth. We used next-generation short-read sequencing data to study this correlation with two $X$. fastidiosa strains causing Pierce's disease (strains STL and Temecula1) grown on a rich-growth solid medium (PWG) for 1 week. Samples were collected daily (except for day 5) and submitted for Illumina sequencing (three biological replicates per strain per day). The mean coverage depth was approximately $600 \times$ for each sample. Changes in the depth of coverage in $10-\mathrm{kbp}$ windows across the genome sequence length showed multiple peaks and troughs (Fig. 2). The origin and the terminus of replication were identified by fitting a linear function to filtered and $\log 2$-transformed depth of coverage values (Brown et al. 2016). A regression of the sequencing depth led to a single peak near the origin of replication and a single trough near the predicted terminus for both strains during the growth phase, while a flat sequencing coverage depth line was observed during the stationary phase (Fig. 2A). No positive correlation was obtained between the computed PTR values and the measured growth rate-as calculated by Korem et al. (2015) (see Materials and Methods) — for either strain ( $P$ value $\geq 0.63)$.

Several small peaks and troughs along the $X$. fastidiosa genome were reproducibly obtained whatever repetition, strain, and time point was considered (Fig. 2B). These variations of coverage depth were positively correlated with the GC content of sequences (Supplementary Fig. S1).

\section{PTR predicts abundance changes} of $X$. fastidiosa when using qPCR data.

Because qPCR is often used to determine bacterial populations, we tested whether this technique could determine PTRs and predict $X$. fastidios $a$ growth rate. Three primer pairs designed at the origin of replication ( $\mathrm{RecF} 1, \mathrm{RecF} 2$, and gyrB) as well as three primer pairs designed at the predicted terminus
(TatD, RuvA, and dif) were tested and the nine different ratio (PTR) combinations were calculated. The best correlations between PTR and growth rate were obtained when using a lag time of 1 day for both strains (Supplementary Tables S1 and S2). In other words, PTRs preceded and foresaw growth rate (GR) with a lag phase of 1 day: $G R_{i+1}=P T R_{i}$, where $G R_{i+1}$ represents the growth rate from day $i$ to day $i+1$. As a consequence, hereafter, we refer to the correlations between PTR at day $i$ and growth rate from day $i$ to day $i+1$, unless otherwise stated. Out of the nine combinations of primer pairs tested, five led to a good correlation between PTRs and growth rate for strain STL (Supplementary Table S2), with RecF2/TatD leading to the best model fit $\left(R^{2}=0.81, P\right.$ value $\left.=0.0004\right)$. Likewise, for strain Temecula1, seven of the nine couples of primer pairs led to a good correlation between both parameters (Supplementary Table S2).

PTR values ranged between 0.8 and 2.2 , with the exception of a PTR $>4$ for strain Temecula1, obtained at day 1 for the three repetitions when using dif as a primer pair for the terminus (Fig. 3). The higher ratios observed for strain Temecula1 during the exponential growth phase in comparison with those observed for strain STL agreed with the estimated shorter generation time for strain Temecula1 (13 h) than for strain STL (18 h) in the growth conditions used.

\section{Growth medium does not alter the correlation between PTR and growth rate.}

Because growth medium affects bacterial growth (Campañharo et al. 2003), its effects on the correlation between PTR and growth rate were tested using the widely employed liquid medium PD2 (Davis et al. 1980) and strain Temecula1. The best correlations between PTR and growth rate were again obtained when using a lag time of 1 day (Supplementary Tables S3 and S4). Similar to strain Temecula1 grown on PWG, the use of the primer pairs RecF1/RuvA and RecF2/RuvA to calculate PTR led to a nonsignificant correlation between PTR and the measured growth rate (Supplementary Table S4). In this case, the use of gyrB/RuvA also resulted in the absence of a correlation. However, the six other couples of primer pairs led to positive and significant correlations between PTR and the measured growth rate, further showing that PTR can predict abundance changes when using qPCR data (Supplementary Table S4). Growth rate was best predicted by PTR when using the RecF1 or RecF2 primer pairs at the origin of replication and the dif

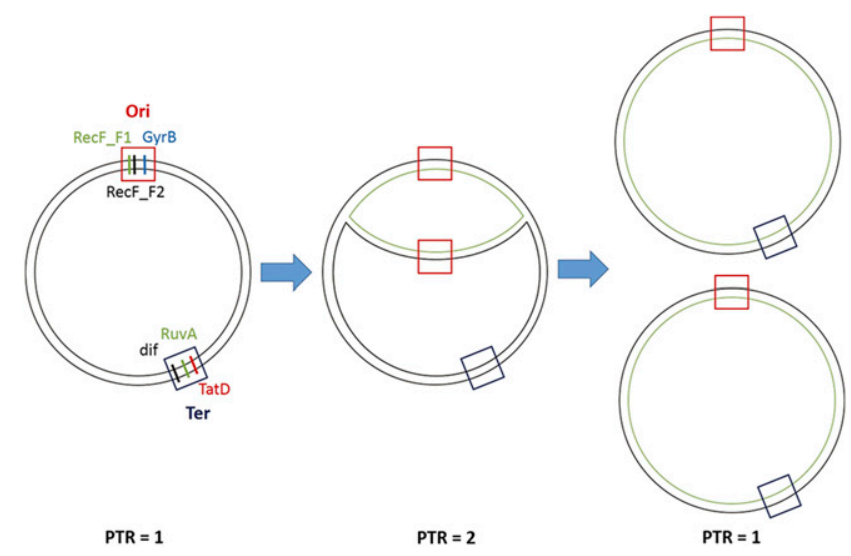

Fig. 1. Bacterial replication and peak-to-trough ratios (PTRs). The red box corresponds to the origin of replication (Ori) while the dark blue box represents the terminus (Ter). Names of the six different primers used in this study to amplify these two regions as well as their approximate locations on the bacterial chromosome are indicated on the first circle. The PTR is shown for each replication step. Parental strands are represented in black while the newly synthetized one is in green. 
primer pair at the terminus of replication $\left(R^{2}>0.4, P\right.$ value $\leq 0.002$ ) (Supplementary Table S4). All of the PTR values ranged between 0.8 and 1.7 (Fig. 4A).

\section{Propidium monoazide treatment helps discriminate between growing and nongrowing $X$. fastidiosa cells.}

We tested whether a photoreactive DNA-binding dye propidium monoazide (PMAxx), which differentiates viable and nonviable $X$. fastidiosa cells (Sicard et al. 2019), could better discriminate between growing and nongrowing $X$. fastidiosa cells. This method relies on the penetration and subsequent intercalation of the photoreactive dye within double-stranded DNA which, upon light exposure, covalently binds to DNA, preventing its amplification by qPCR. Because this dye is able to penetrate cells with damaged membranes only, it is assumed to exclusively prevent DNA amplification from these cells. The qPCR-based PTR estimate corresponds to the average ratio calculated with all of the cells (i.e., template copies) in a sample, including nonviable cells. Dead cells should have a PTR of 1, decreasing the average ratio of a sample if viable cells are replicating while having no significant effect on nongrowing populations. As a consequence, the presence of dead cells should create a systematic bias in PTR estimates and make it more difficult to distinguish between growing and nongrowing populations. Although PMAxx treatment did not have a large impact on the PTR values obtained for six of the nine combinations of primer pairs used (data not shown), a 10fold difference was observed between the PTR of populations in exponential and stationary phases when the dif primer pair near the predicted terminus was used. This led to a ratio between 1.15 and 2.6 when cells were in the exponential phase and an average ratio between 0.06 and 0.15 during stationary phase (Fig. 4B). The resulting PTR values were correlated with the measured growth rate $\left(R^{2} \geq 0.66, P\right.$ value $\left.=0.0002\right)($ Fig. 4C; Supplementary Table S4).

\section{Can PTR report $X$. fastidiosa growth status}

\section{in plants or insects?}

We tested whether PTR could be used to estimate $X$. fastidiosa population growth in plant and insect hosts using the PMAxx-qPCR protocol and the RecF1 and dif primer pairs. Leaf petioles from mechanically inoculated grapevines were sampled at different times postinoculation and at different distances above the inoculation point. Because different plants were sampled at the three times postinoculation (no repeated measures, disruptive sampling), the measured growth rate could not be calculated. Instead, we generated the viable cell population using PMAxx-qPCR (Sicard et al. 2019) and looked at the relationship between $X$. fastidiosa population size and PTR. We expected a priori that the relationship between $X$. fastidiosa population size and PTR may be unimodal—with increasing population size and PTR increasing up to a peak, then declining again. Our results fit our predictions of a unimodal relationship in plants (Fig. 5A), with the Holling Type IV model fitting the data significantly better than a linear model $\left(\Delta \mathrm{AIC}_{\mathrm{c}}=1.04 \times\right.$ $10^{6}$ ). Parameter estimates of the Holling Type IV model were well constrained, with narrow 95\% confidence intervals (Supplementary Table S5). Based on our parameter estimates, peak population size occurred at a PTR value of 1.01. These results suggest that localized and large $X$. fastidiosa populations within plants are no longer multiplying. Interestingly, for lower and higher PTR values-PTR $<0.75$ and PTR $>1.25$, respectively (Fig. 5A) - the viable cell population was very low (viable cell population $<4.92 \times 10^{3} \mathrm{CFU} / \mathrm{g}$ ). Although a low viable cell population combined with a low PTR value suggests that the bacteria are not dividing, a higher PTR value indicates that at least part of the bacterial population is actively growing, such as a population expanding in size in a newly colonized xylem vessel. The latter is expected for $X$. fastidiosa, given that growth within plants is heterogeneous and limited by xylem vessel structure (Chatterjee et al. 2008). This observation is of epidemiological relevance because it suggests that the knowledge of both PTR and cell population size can provide insights into the bacterial cell growth in planta.

Similarly, we tested whether PTR could predict changes in cell abundance in an insect vector (Graphocephala atropunctata). G. atropunctata individuals were collected from noninfected basil plants at different times following acquisition from $X$. fastidiosa-infected plants. Both the bacterial-viable population and PTRs were assessed for each individual. PTR values ranged between 0.11 and 1.62. As in the in planta experiment, the Holling Type IV model fit the data from $G$. atropunctata significantly better than a linear model $\left(\Delta \mathrm{AIC}_{\mathrm{c}}=\right.$ 574.3). Our parameter estimates indicate that peak population size occurred at a PTR value of 1.16 but the peak was much less clear compared with the in planta experiment (Fig. 5B) (Supplementary Table S5). These results are further discussed below.
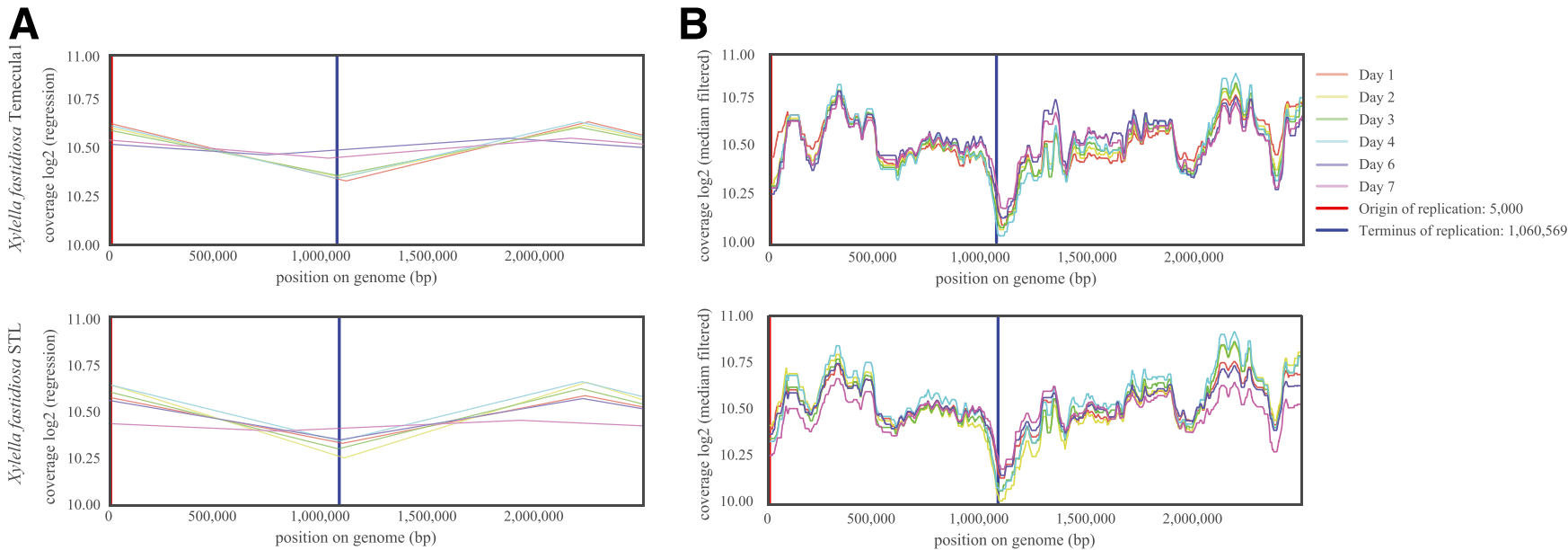

Fig. 2. Genome coverage depth graphics of Xylella fastidiosa subsp. fastidiosa Temecula1 (above) and STL (below) across time. A, Regression of the sequencing coverage depth led to a single peak near the origin of replication and a single trough near the predicted terminus during the first days of growth while a quasi-uniform coverage across the genome was observed at later time points. B, Median filtered coverage shows the coverage variations along the genome. Three biological replicates have been pooled. 


\section{DISCUSSION}

Quantitative PCR is arguably the current method of choice to estimate bacterial population growth (Bustin et al. 2009). Although useful, this measurement provides static information on populations and does not reflect the immediate dynamics of a population without multiple sampling periods. We showed that

A

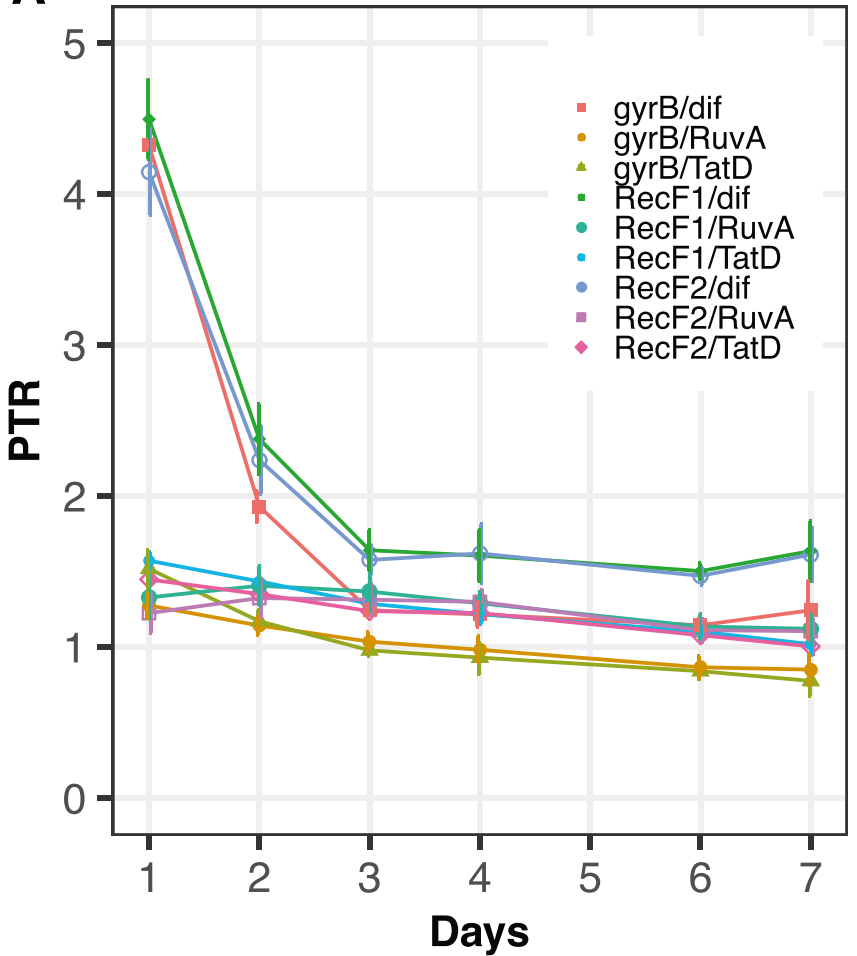

C

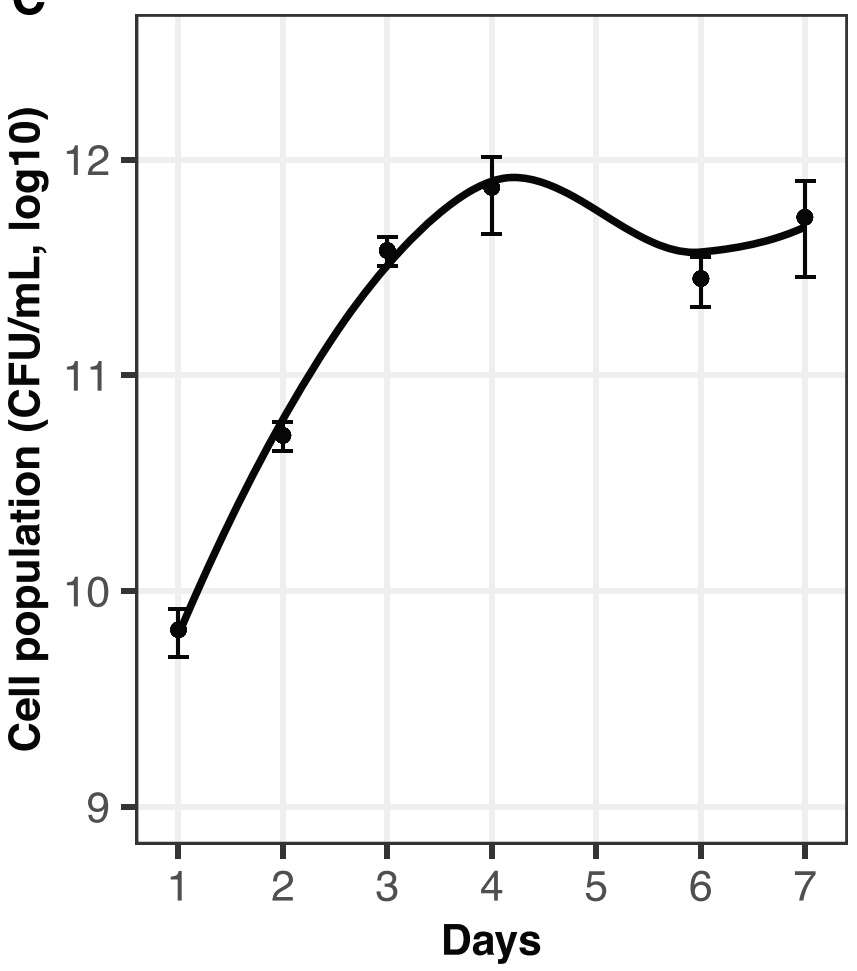

qPCR may provide information on bacterial growth status for a slow-growing plant pathogen using a single time point if two primer pairs are used at opposite regions of the chromosome, one near the origin and another at the terminus of replication.

We observed no correlation between PTR and the measured growth rate when using next-generation sequencing data. The origin of replication and terminus positions calculated by the

B

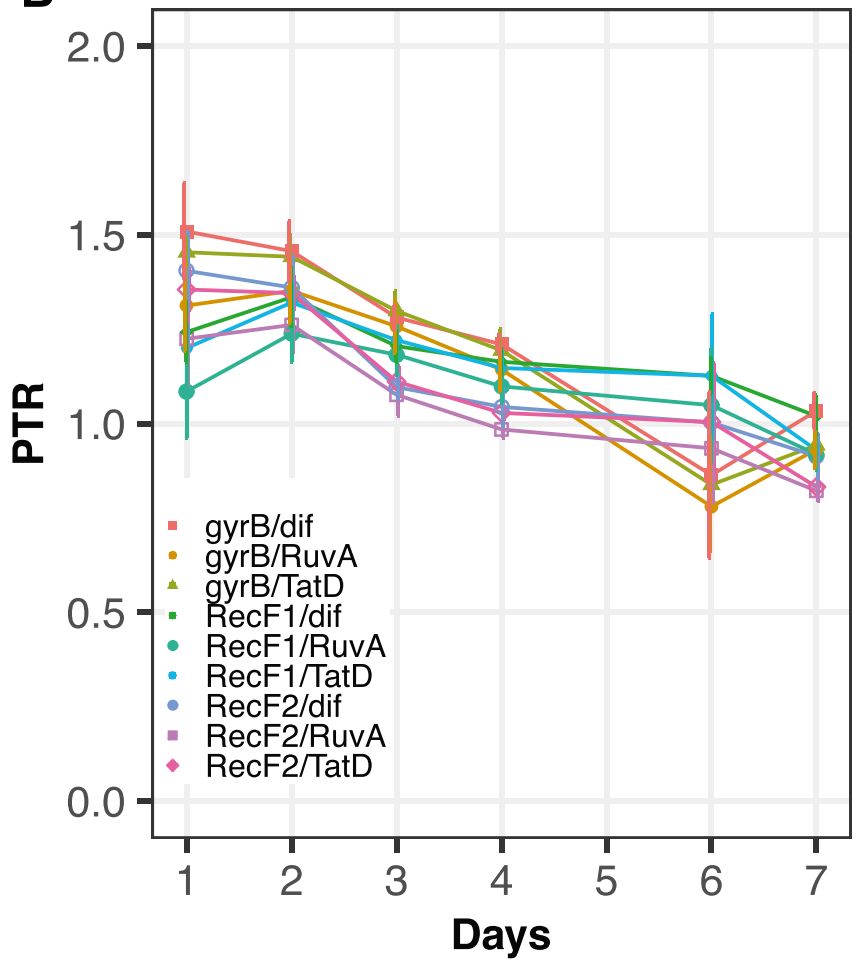

D

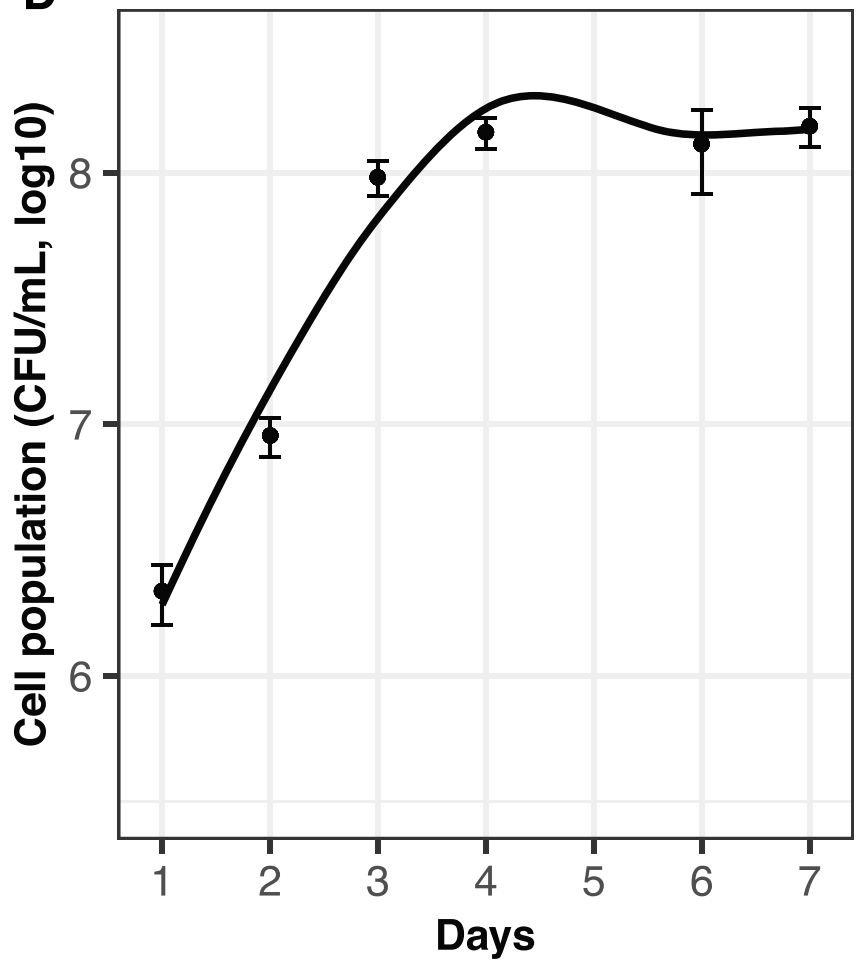

Fig. 3. Peak-to-trough ratios (PTRs) predict Xylella fastidiosa abundance changes when using quantitative PCR data. PTRs across time of strains A, Temecula1 and B, STL (ratios obtained using the nine couples of primer pairs are shown); $\mathbf{C}$ and D, display their growth curves, respectively. Mean and standard errors based on the analyses of three replicates are displayed. Both strains were grown on the solid medium PWG. 
software developed to estimate PTR from metagenomic data (Brown et al. 2016) led to PTR values more abridged than expected based on the graphs and did not correctly reflect the changes in PTR across time. This discrepancy could explain the

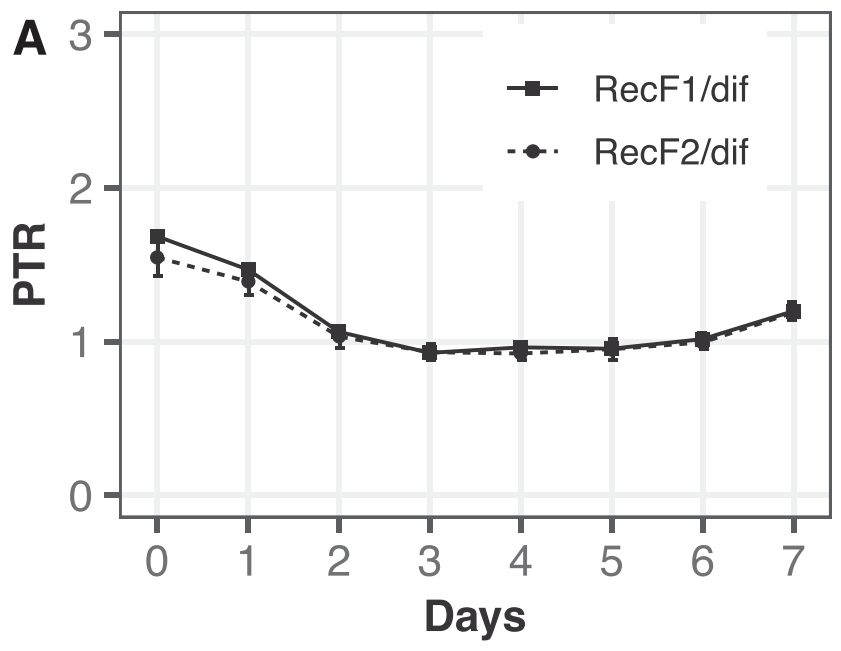

B
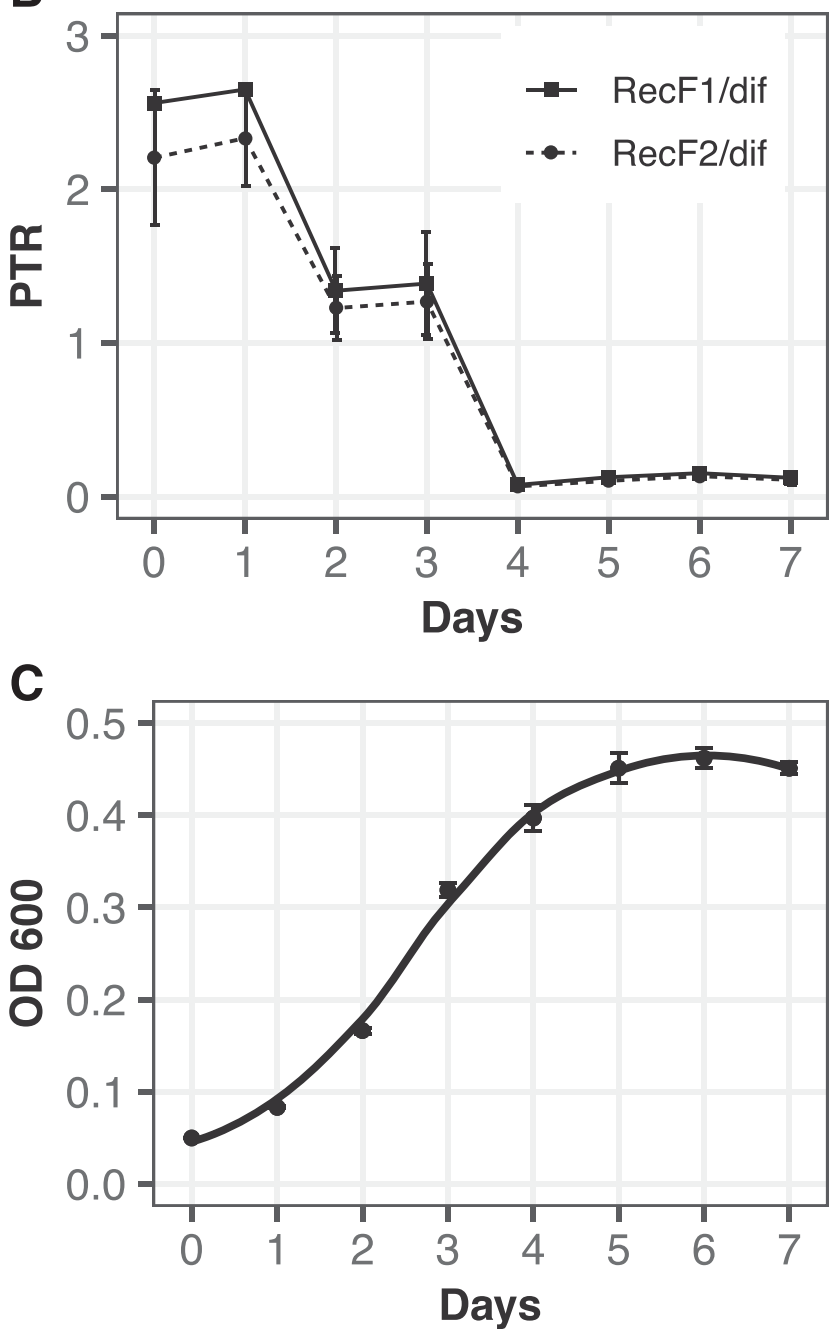

Fig. 4. Propidium monoazide (PMAxx) helps discriminate between growing and nongrowing Xylella fastidiosa Temecula1 cells grown in the liquid medium PD2. PTRs obtained A, without and B, with PMAxx pretreatment are correlated with $\mathbf{C}, X$. fastidios $a$ measured growth rate. $\mathrm{OD}_{600}=$ optical density at $600 \mathrm{~nm}$. Mean and standard errors based on the analyses of three replicates are displayed. During stationary phase, PTRs between 0.06 and 0.15 were obtained. absence of correlation here obtained. Even though numerous peaks and troughs were observed along the length of the genome, the expected trend of more template near the origin of replication in relation to the terminus was observed at the genome level, even if subtly. These coverage variations were only partially explained by variation in the percentage of GC across the length of the genome; a trend which has been well documented (Benjamini and Speed 2012; Dohm et al. 2008).

When the same samples were processed with qPCR, PTR values were significantly and positively correlated with the measured growth rate. The highest correlations were obtained
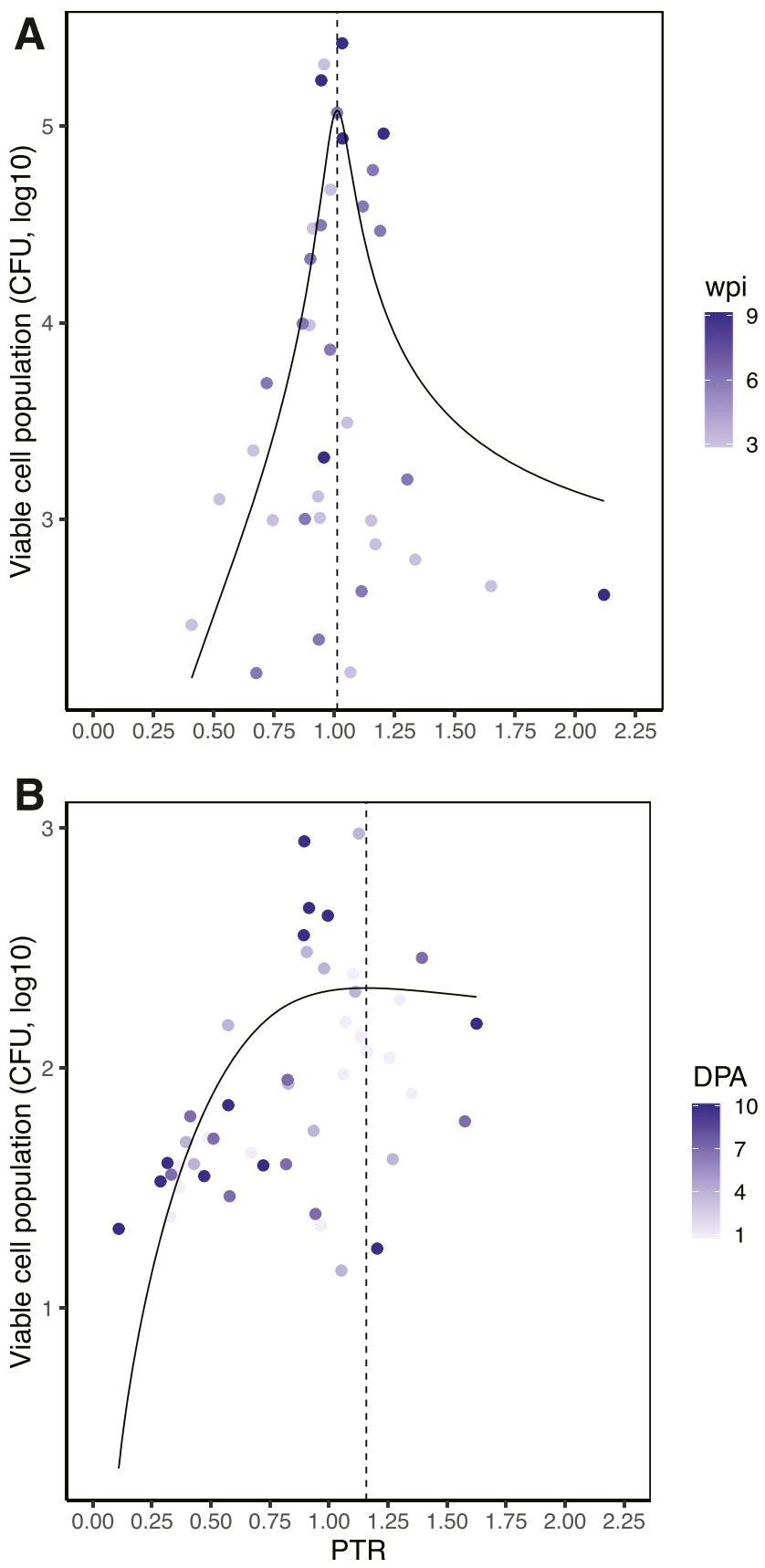

Fig. 5. Relationship between peak-to-trough ratio (PTR) and viable cell population size of Xylella fastidiosa estimated in $\mathbf{A}$, grapevines and $\mathbf{B}$, Graphocephala atropunctata vectors. Solid lines indicate the predicted fit of the Holling Type IV model to the data. Colors of points indicate weeks postinoculation (wpi) for grapevines or days since acquisition (DPA) for $G$. atropunctata, with darker colors representing later time points. Viable cell populations were $\log 10$ transformed. 
when using a lag phase of 1 day, suggesting that PTRs precede and foresee $X$. fastidiosa population growth by 1 day at least in vitro. This was true for the two strains tested, the two-growth media used, and whether cells were or were not treated with PMAxx. The different doubling times calculated for each in vitro experiment in order to measure the growth rate $(16 \mathrm{~h}$ for strain STL on PWG, and 13 and $9 \mathrm{~h}$ for strain Temecula1 grown on PWG and in PD2, respectively) were in the range of the values previously reported for $X$. fastidiosa cells (Feil and Purcell 2001; Guilhabert and Kirkpatrick 2005; Smolka et al. 2003). The absence of a codon usage bias toward highly expressed genes has been suggested to be at the origin of the slow growth of this bacterium (Smolka et al. 2003). The preferential use of given codons over synonymous ones in coding DNA, especially in highly expressed genes, which is correlated with a bias in abundance of matching tRNA, is indeed assumed to optimize the cell growth efficiency (Kurland 1991). Smolka et al. (2003) observed very small codon bias for some highly expressed $X$. fastidiosa genes, as well as a similar codon bias distribution for all of the genes regardless of their expression profile. The low nutrient content in the xylem, where $X$. fastidiosa is restricted, may also be linked to its slow-growing nature (Purcell and Hopkins 1996; Smolka et al. 2003). Lastly, $X$. fastidiosa has two ribosomal RNA operons (while Escherichia coli has seven copies per genome, for example) and the number of operons has been positively correlated with growth rate (Klappenbach et al. 2000).

Two origin/terminus (Ori/Ter) pairs of primer pairs, namely RecF1/dif and RecF2/dif, reproducibly led to a good correlation between the PTRs and the measured growth rate in spite of medium, strain, and treatment considered. RecF1 and RecF2 both target the same gene $(R e c F)$, which encodes a DNA replication and repair protein. When using a primer pair targeting another gene at the origin of replication, $g y r B$, encoding for the subunit B of the DNA gyrase, and dif primers at the terminus, the same trends were observed. The PTRs obtained using this primer pair (gyrB/dif) were also correlated with the measured growth rate for the three in vitro experiments with strain Temeculal but the correlations between PTR and growth rate were marginally significant when using strain STL. Although the six other Ori/Ter couples of primer pairs tested led to a significant correlation between PTR and $X$. fastidiosa abundance changes for some of the in vitro experiments, we found that, when using these primer pairs, the significance of these correlations depended on the experimental conditions. Therefore, we favored the use of RecF1 and dif primer pairs to run the in vivo experiments.

Overall, these correlation results show that, although the primer pairs used targeted the same regions of the genome (either the origin of replication or the terminus), differences of ratios could be observed, which eventually led to different correlation values. In fact, although the trends were similar for the different PTRs computed, with a global decrease of the ratios during the growth exponential phase and a relative constant ratio averaging 1 during the stationary phase, the absolute values of the ratios differed. For the three replicates of strain Temecula1 cells grown on PWG, we observed a ratio $>4$ at day 1 when using the dif primer pair for the terminus regardless of the primer pair used for the origin of replication. Such a high ratio could be interpreted as the result of multiple replication forks. However, the ratios between 1.23 and 1.57 observed at the same time point when using other Ori/Ter primer pairs seem to refute this assumption. Furthermore, ratios between 1.15 and 1.64 , instead of a ratio of 1 , were obtained during the stationary phase of this same experiment when using the dif primer pair at the terminus, suggesting that the interpretation of the ratios might not always be straightforward. The impact of the primer pairs on PTRs was even more pronounced when samples were treated with PMAxx (data not shown). Therefore, although all Ori/Ter couples of primer pairs should theoretically result in similar ratios, primer pairs affected PTR values significantly.

It is worth mentioning that, although the primer pair at the origin influences the ratio obtained, the primer pair at the terminus appears to influence PTR estimates more strongly. These differences of ratios between Ori/Ter primer pairs are likely not due to differences of amplification efficiency, because these were taken into account by using the LinReg software (Ruijter et al. 2009). In addition, all of the genes targeted by our primers have a single copy within the $X$. fastidiosa genome; this was checked in silico, by PCR, and by analyzing the qPCR melting curves. However, differences of GC content were observed among the amplified regions, with the lowest GC content detected in the region amplified by the dif primers (36.4\%). Comparatively, other amplified regions displayed a GC content ranging between 44 and $51 \%$. Interestingly, we noticed that the dif region also presented a drop of sequencing coverage depth.

Because qPCR leads to the amplification of intact DNA template, regardless of cell viability, we tested whether the use of a viability dye such as PMAxx could help better discriminate between samples containing dividing cells with those containing nondividing cells. Indeed, the PTR of dead cells should be approximately 1 because the number of copies at the origin should equal the one at the terminus of replication in the absence of preferential DNA degradation. Thus, the presence of dead cells within a sample should not have a significant effect on the PTR of samples containing cells in a stationary phase while decreasing the average ratio of a sample containing dividing cells. The effect on the later should increase with the proportion of dead cells within the sample. Although the use of PMAxx did not increase the ratio difference between dividing and nondividing cells when using either TatD or RuvA primers for the terminus (data not shown), a 10-fold difference was observed when using dif primers. This difference is critical because it can be difficult to conclude whether cells are dividing or not based on a ratio difference of 1.3- to 2-fold, as we mostly observed in vitro in the absence of a PMAxx pretreatment for this slow-growing bacterium. This small difference of ratio, in the absence of a pretreatment, might be even lower in vivo, where cells are growing asynchronously and present a lower doubling time. Interestingly, the increase of ratio of 1 (on average, PTR = 1.55 for nontreated cells and PTR $=2.47$ for PMAxx-treated cells) observed at the beginning of the exponential phase (days 0 and 1), when samples from this experiment were treated with the viability dye PMAxx, suggest that a proportion of the cells collected in the stationary phase to start the cultures might have been dead, thus lowering the PTR in the absence of a PMAxx pretreatment. Although we cannot explain the drop of PTR in the stationary phase to values between 0.06 and 0.15 , the obtained ratios were even better correlated to the measured growth rate compared with when the samples were nontreated. Based on these data, for example, a ratio $>2$ could indicate that most cells are growing whereas a ratio $<0.15$ would signal that most cells are no longer dividing; thus, intermediate values would correspond to different proportions of dividing cells within a sample. Further experiments using cells growing synchronically should help confirm this assumption.

These results led us to run our in vivo samples using PMAxxqPCR and the RecF1 and dif primer pairs. Because $X$. fastidiosa multiplies in both its plant hosts and its insect vectors, we examined the distributions of PTR and viable cell populations in both grapevines and blue-green sharpshooters (BGSS). The absence of repeated measures - caused by the destructive sampling - and the absence of a known generation time for the in vivo experiments prevented us from calculating the measured growth rate in vivo. Generation times or doubling times are indeed difficult to assess in 
vivo (Gibson et al. 2018), mainly because of the lack of appropriate data and tools to directly measure them.

The period over which $X$. fastidiosa cells multiply within their plant hosts and their vectors remains mostly unknown. Nevertheless, based on previous data (Killiny and Almeida 2009; Labroussaa et al. 2017), we hypothesized that the multiplication should mainly occur during the first week following the acquisition of bacterial cells in insects. Similarly, based on the slow growth of $X$. fastidiosa in grape and previous data (A. R. Zeilinger, C. Wallis, D. J. Beal, A. Sicard, A. Walker, and R. P. P. Almeida, unpublished data), we decided to sample petioles between 3 and 9 weeks postinoculation. In both insects and plants, we observed a unimodal relationship between $X$. fastidiosa viable cell population size and PTR with a peak near 1 (peak in grapevines $=1.01$; peak in $G$. atropunctata $=1.16$ ). The PTRs obtained in vivo span the same range of values as in vitro, suggesting that the values obtained in vitro are not linked to specific experimental conditions and their interpretation regarding $X$. fastidiosa growth status might be applied in vivo. Population size of $X$. fastidiosa within hosts is epidemiologically relevantpopulation size in both grapevines and vectors being positively associated with vector transmission (Zeilinger et al. 2018). The combination of viable cell population size and PTRs should provide even greater insights into the cell population dynamics, by providing both the population size (and, thus, the relative probability of transmission) at the time of sampling and a prediction of population size in the immediate future.

The absence of a clear peak population size in G. atropunctata could be explained by the short period analyzed in this study, which led to smaller bacterial populations than usually observed. This vector species has been shown to harbor up to $10^{5} \mathrm{X}$. fastidiosa cells per insect (Killiny and Almeida 2009; Rashed et al. 2011). Because we focused on the 10 first days following acquisition, it is possible that we did not span the entire multiplication period of $X$. fastidiosa within its vector, resulting in these lower bacterial population sizes (up to $10^{3} \mathrm{CFU} /$ insect). Moreover, because the experiment was conducted with mature adults, we cannot exclude that the physiological conditions of these insects might have been suboptimal for $X$. fastidiosa growth. Further experiments using longer periods and young adults may result in a clearer relationship between PTR and bacterial populations within insects.

We show that qPCR data can provide both a static measurement of bacterial abundance and a dynamic view of its abundance changes by calculating PTR, and that this concept may be applied to slow-growing bacterial species both in vitro and in vivo, although further work will help confirm our in vivo results. The sequencing depth of coverage trends observed in this study suggest that short-read high-throughput data may not be useful to infer PTR for slow-growing bacteria, although that should be explored with other taxa. Furthermore, we expect that the multiple peaks and troughs observed in depth of coverage for $X$. fastidiosa have evolutionary meaning and a biological role, even if we were not able to speculate on those in this study. Ultimately, PTR estimates using sets of primer pairs at the origin and terminus of the chromosome could be used to determine the effect of biotic and abiotic stressors on the growth rate of other plant-associated bacteria. Moreover, because qPCR can be species or even strain specific, qPCR-PTR could be used to determine growth status within bacterial communities.

\section{MATERIALS AND METHODS}

\section{Strains and media.}

$X$. fastidiosa subsp. fastidiosa STL and Temecula1 were used throughout this study. The cells were grown either on the solid medium PWG (Hill and Purcell 1995) or in $5 \mathrm{ml}$ of the liquid medium PD2 (Davis et al. 1981) under constant agitation at
$150 \mathrm{rpm}$ at $28^{\circ} \mathrm{C}$. For the first in vitro experiment, both strain Temecula1 and STL cells were grown on the solid medium PWG for 1 week-the time at which cells had reached the stationary phase- - before being resuspended into succinate-citratephosphate buffer (SCP) (Hopkins 1984) at an optical density at $600 \mathrm{~nm}\left(\mathrm{OD}_{600}\right)$ of 0.3. Three suspensions - corresponding to our three replicates - for each strain were prepared and replated on six PWG plates for the time-course experiment. All of the cell scrapings from one PWG plate for each strain and for each replicate were collected at $1,2,3,4,6$, and 7 days postplating and resuspended in $1 \mathrm{ml}$ of SCP.

For the second in vitro experiment, strain Temecula1 cells were grown on PWG plates for 7 days before being collected and resuspended in PD2 medium at an initial $\mathrm{OD}_{600}$ of 0.05 . Three different suspensions were prepared and divided into eight vials of $5 \mathrm{ml}$ each. At each time point-corresponding to every day starting from day 0 (i.e., from the resuspension step to day 7) - two fractions of $500 \mu \mathrm{l}$ each were collected per replicate $(n=3)$. One fraction was treated with $25 \mu$ M PMAxx as previously described (Sicard et al. 2019) while the other fraction remained untreated.

\section{Plant and insect sampling.}

Two-month-old Vitis vinifera 'Cabernet sauvignon' plants were pinprick inoculated with $10 \mu \mathrm{l}$ of an inoculum containing $X$. fastidiosa STL cells resuspended in SCP at a concentration of $10^{8} \mathrm{CFU} / \mathrm{ml}$. In total, 37 grape petioles were sampled at 3,6 , and 9 weeks postinoculation at four different distances from the inoculation point (first petiole below the inoculation point and first, fourth, and sixth petioles above it). All of the petioles were sterilely sliced before being homogenized using a Polytron (Brinkman Instruments, Inc., Westbury, NY, U.S.A.), as previously described (Hill and Purcell 1995). The homogenate was divided into two: one part was treated with $25 \mu \mathrm{M}$ PMAxx, as previously described (Sicard et al. 2019), while the other part remained untreated before being further processed.

BGSS ( $G$. atropunctata) were reared from nymphs in our greenhouse facility at the University of California-Berkeley as previously described (Zeilinger et al. 2018). After 1 day of acquisition on $X$. fastidiosa-infected grape with strain STL, the insects were transferred to healthy basil plants. Insects were sampled at 1, 4, 7, and 10 days after the end of acquisition. Between 9 and 18 insects were sampled per time point (47 insects in total). Insect heads were cut before being homogenized into SCP buffer. As described above, each sample was divided into two, one part being PMAxx-treated while the other remained untreated.

\section{DNA extraction.}

DNA from pure culture grown on PWG plates and from G. atropunctata heads was extracted using the blood and tissue kit (Qiagen) and preceded by a pretreatment for Gram-negative bacteria, as suggested in the manufacturer's instructions. DNA from cells grown in PD2 was extracted following a modified cetyltrimethylammonium bromide protocol (Doyle and Doyle 1987) while DNA from grape petioles was extracted using the DNeasy plant mini kit (Qiagen).

\section{Coverage depth and replication analysis using next-generation sequencing data.}

$X$. fastidiosa subsp. fastidiosa Temecula1 and STL cells collected from PWG plates at days 1, 2, 3, 4, 6, and 7 were sequenced via Illumina HiSeq 4000 at the University of California-Berkeley Vincent J. Coates Genomics Sequencing Laboratory (California Institute for Quantitative Biosciences). All raw reads and information regarding each sample have been submitted to the Sequence Read Archive (SRA) database (SRA 
accession PRJNA521404). The total number of FASTQ reads for each strain and day of the experiment varied across replicates. Seqtk 1.2 was used to normalize variation in the raw data by obtaining a subsample of $n=6,274,589$ reads within each forward FASTQ file, where $N$ represents the smallest number of reads in a single FASTQ file used in the study. A publicly available script (mergeShuffledFastqSeqs.pl) was used to synchronize forward and reverse FASTQ files for each set of paired data. Normalized reads were then mapped to strain Temecula1 (ASM724v1) with Bowtie2 (Langmead and Salzberg 2012) using the no-unal and reorder parameters.

The resulting SAM files were converted into BAM, indexed, and sorted using Samtools 1.8 (Li et al. 2009). Following this, depth for each strain and day pair was obtained using the BamCoverage function of Deeptools 2.0 (Ramírez et al. 2016) using a bin size of $20 \mathrm{bp}$ and normalized using the reads per kilobase million method. Coverage depth across the length of the $X$. fastidiosa chromosome was visualized using the EPIC$\mathrm{CoGe}$ tool on the CoGe web-based platform. In addition, the relationship between variation in depth and differences in local nucleotide composition were evaluated using a linear regression and Pearson correlation test in Rstudio, version 1.1.456 (Supplementary Fig. S1). Additionally, bacterial replication rates were measured in each strain for the duration of the experiment using a modified bPTR script (Brown et al. 2016). Briefly, bPTR was run recursively on all SAM files corresponding to each strain using the pickle parameter, which allows their serialization. A modified bPTR script, also provided by Brown, was then used to compile bPTR calculations and visualize them in a single figure (Fig. 2).

\section{Primers design and qPCR.}

Two primers pairs targeting the gene encoding for the DNA replication and repair protein $\operatorname{RecF}$ (RecF, PD0003), namely RecF1_F+R and RecF2_F+R, hereafter abbreviated RecF1 and $\mathrm{RecF} 2$, and one primer pair targeting the gene encoding for the DNA gyrase subunit B ( $g y r B$, PD0005) were designed and tested in silico. Three different primer pairs were also designed in the terminus region and tested in silico; one primer pair targeting the gene encoding for a preprotein translocase subunit TatD (PD0878), one within the gene encoding for a Holliday junction branch migration protein RuvA (PD0887), and one primer pair amplifying the region near the predicted terminus, dif (Kono et al. 2011). All of the primer pairs are named in the text in reference to the region of the gene they are amplifying; namely RecF1, RecF2, gyrB, TatD, RuvA, and dif. The regions amplified by the six primer pairs were present only once within the genome of both strains. The names, genomic positions, and efficiencies of the primer pairs are summarized in Supplementary Table S6. A schematic representation of their positions can also be viewed in Figure 1. Different concentrations were tested for each primer pair to define their optimal concentration in our conditions; gyrB and RuvA were used at a final concentration of $0.15 \mu \mathrm{M}$; RecF1, RecF2, and dif were used at a final concentration of $0.3 \mu \mathrm{M}$; and TatD was used at a final concentration of $0.5 \mu \mathrm{M}$. All of the qPCRs were run on a 7500 Fast Real-Time PCR thermocycler using the GoTaq qPCR Master Mix (Promega Corp.) and the following conditions: 40 cycles of $95^{\circ} \mathrm{C}$ for $10 \mathrm{~s}, 63^{\circ} \mathrm{C}$ for $15 \mathrm{~s}$, and $72^{\circ} \mathrm{C}$ for $30 \mathrm{~s}$. Each sample was run in duplicate. Fluorescence data were normalized using the reference dye provided within the kit and analyzed using the LinRegPCR program, which takes into account primer pair efficiency (Ruijter et al. 2009). Bacterial populations were calculated using a standard curve of the proper bacterial strain and the proper pair of primers. The PTRs were calculated by dividing the copy number at the origin of replication by the copy number at the terminus for each couple of primer pairs. The growth curves of the samples grown on solid medium were obtained by using qPCR data and the dif primer pair; the bacterial population in CFU per gram was calculated by using the proper standard curve and the amount of plant material analyzed. The correlations between PTRs and the measured growth rate were slightly improved when using the primer pair at the origin of replication, $\mathrm{RecF} 1$, to calculate the bacterial population (instead of dif). However, this improvement was only marginal and did not affect the conclusions presented here. $\mathrm{OD}_{600}$ was measured for $X$. fastidiosa cells grown in a liquid medium at each sampling time using a spectrophotometer (Genesys 10S Vis; Thermo Fisher).

\section{Statistical analyses.}

The specific growth rate and the generation time were calculated for each strain and each growth medium using the following equations (Maier and Pepper 2015):

$$
\begin{gathered}
(d X / d t)=\mu X \\
T=(\ln 2 / \mu)
\end{gathered}
$$

where $X$ is the bacterial relative abundance, $t$ is time, $\mu$ is the specific growth rate, and $T$ is the generation time. Measured growth rates were calculated according to the equation described by Korem et al. (2015) with and without a lag time of 1 day. Growth without a lag period was calculated as:

$$
G R_{i}=\log 2\left[\left(X_{i} / X_{i-1}\right) /\left(t_{i}-t_{i-1}\right)\right] \times 1 / T
$$

where $G R_{i}$ represents the growth rate from time $i-1$ to time $i$. Similarly, growth rate with a lag period was calculated as:

$$
G R_{i+1}=\log 2\left[\left(X_{i+1} / X_{i}\right) /\left(t_{i+1}-t\right)\right] \times 1 / T
$$

where $G R_{i+1}$ represents the growth rate from time $i$ to time $i+1$.

Using measured growth rate both with and without a time lag, we tested for a relationship with $P T R_{i}$ (i.e., PTR at time $i$ ) and growth rate using linear regression. We used permutation tests to calculate one-tailed $P$ values following Korem et al. (2015) due to violation of linear model assumptions. Parametric $P$ values largely corresponded to permutation $P$ values (data not shown).

We evaluated the relationship between PTR and growth rate using different qPCR primer pairs using both Aikake's Information Criterion (AIC) and $R^{2}$. Although $R^{2}$ is generally a poor measure of how a model fits to data because it lacks a penalty against model complexity (i.e., number of parameters) (Bolker 2008), all models in our analyses were of the same complexity. As a result, the rankings of primer pairs based on $R^{2}$ corresponded closely to rankings using AIC (Supplementary Tables S1 to S4).

We analyzed the relationship between PTR and growth in vivo from BGSS vectors and grapevine plants using populations of $X$. fastidiosa cells rather than growth rate. Because of destructive sampling in both cases, we could not collect repeated measures of $X$. fastidiosa populations. Because of an a priori expectation that the relationship between PTR and $X$. fastidiosa population size would be unimodal, we fit the data to the Holling Type IV function response model:

$$
y=a x^{2} /\left(b+c x+x^{2}\right)
$$

where $y$ represents $X$. fastidiosa population size, $x$ represents PTR value, and $a, b$, and $c$ are parameters to be estimated. We then fit the Holling Type IV model and a linear model to the data using a maximum-likelihood estimate and compared model fits with $\mathrm{AIC}_{\mathrm{c}}$. For data from BGSS, all data across time points postinoculation were fit to the same model, and we treated data from grapevines similarly-fitting one model to all 
data across distances from the inoculation point. Confidence intervals were calculated for parameter estimates using quadratic approximation (for more details on the equations and model fitting process, see A. R. Zeilinger, C. Wallis, D. J. Beal, A. Sicard, A. Walker, and R. P. P. Almeida, unpublished data, and Bolker 2008). Based on the parameter estimates of the Holling Type IV, we calculated the PTR value corresponding to peak $X$. fastidiosa population size as $-2 b / c$ (Bolker 2008).

In all analyses, PTR was centered at 0 by subtracting the mean, and $X$. fastidiosa populations (when used in lieu of growth rate) were $\log 10$ transformed. All analyses were performed in R 3.5.2 (R Core Development Team 2018), with the exception of the Spearman correlation test that was run using JMP (version 14; SAS Institute Inc., Cary, NC, U.S.A.). Permutation tests were run using the permuco package (Frossard and Renaud 2019). Nonlinear model fitting was conducted using the bbmle package (Bolker and $\mathrm{R}$ Core Team 2017).

\section{ACKNOWLEDGMENTS}

We thank D. J. Beal for rearing and providing the insects for this study and the Foundation Plant Services at Davis (Davis, CA, U.S.A.) for providing the grapevines.

\section{AUTHOR-RECOMMENDED INTERNET RESOURCES}

CoGe platform: https://genomevolution.org/coge

Seqtk 1.2: https://github.com/lh3/seqtk

\section{LITERATURE CITED}

Almeida, R. P. P., and Nunney, L. 2015. How do plant diseases caused by Xylella fastidiosa emerge? Plant Dis. 99:1457-1467.

Almeida, R. P. P., Pereira, E. F., Purcell, A. H., and Lopes, J. R. S. 2001. Multiplication and movement of a citrus strain of Xylella fastidiosa within sweet orange. Plant Dis. 85:382-386.

Almeida, R. P. P., and Purcell, A. H. 2003. Biological traits of Xylella fastidiosa strains from grapes and almonds. Appl. Environ. Microbiol. 69:7447-7452.

Becerro, S., Paredes, J., and Arana, S. 2015. Multiparametric biosensor for detection and monitoring of bacterial biofilm adhesion and growth Pages 333-336 in: 6th Eur. Conf. Int. Fed. Med. Biol. Eng. I. Lacković and D. Vasic, eds. Springer International Publishing, Cham.

Benjamini, Y., and Speed, T. P. 2012. Summarizing and correcting the GC content bias in high-throughput sequencing. Nucleic Acids Res. 40: e72.

Bolker, B., and R Core Team. 2017. bbmle: Tools for general maximum likelihood estimation. https://rdrr.io/cran/bbmle/

Bolker, B. M. 2008. Ecological Models and Data in R. Princeton University Press, Princeton, NJ, U.S.A.

Brown, C. T., Olm, M. R., Thomas, B. C., and Banfield, J. F. 2016. In situ replication rates for uncultivated bacteria in microbial communities. bioRxiv. doi:10.1101/057992

Bustin, S. A., Benes, V., Garson, J. A., Hellemans, J., Huggett, J., Kubista, M., Mueller, R., Nolan, T., Pfaffl, M. W., Shipley, G. L., Vandesompele, J., and Wittwer, C. T. 2009. The MIQE guidelines: Minimum information for publication of quantitative real-time PCR experiments. Clin. Chem. 55:611-622.

Campañharo, J. C., Lemos, M. V. F., and Lemos, E. G. de M. 2003. Growth optimization procedures for the phytopathogen Xylella fastidiosa. Curr. Microbiol. 46:99-102.

Chatterjee, S., Wistrom, C., and Lindow, S. E. 2008. A cell-cell signaling sensor is required for virulence and insect transmission of Xylella fastidiosa. Proc. Natl. Acad. Sci. U.S.A. 105:2670-2675.

Davis, M. J., French, W. J., and Schaad, N. W. 1981. Axenic culture of the bacteria associated with phony disease of peach and plum leaf scald. Curr. Microbiol. 6:309-314.

Davis, M. J., Purcell, A. H., and Thomson, S. V. 1980. Isolation media for the Pierce's disease bacterium. Phytopathology 70:425-429.

Dohm, J. C., Lottaz, C., Borodina, T., and Himmelbauer, H. 2008. Substantial biases in ultra-short read data sets from high-throughput DNA sequencing. Nucleic Acids Res. 36:e105.
Doyle, J. J., and Doyle, J. L. 1987. Doyle, J., and Doyle, J. 1987. Genomic plant DNA preparation from fresh tissue-CTAB method. Phytochem. Bull. 19:11-15.

European Food Safety Authority (EFSA). 2018. Update of the Xylella spp. host plant database. EFSA J. 16:e5408.

Feil, H., and Purcell, A. H. 2001. Temperature-dependent growth and survival of Xylella fastidiosa in vitro and in potted grapevines. Plant Dis. 85:1230-1234.

Frossard, J., and Renaud, O. 2019. permuco: Permutation tests for regression, (repeated measures) ANOVA/ANCOVA and comparison of signals. R package version 1.0.2. https://cran.r-project.org/web/packages/permuco/index.html

Gallagher, T., Phan, J., and Whiteson, K. 2018. Getting our fingers on the pulse of slow-growing bacteria in hard-to-reach places. J. Bacteriol. 200: e00540-18.

Gfeller, K. Y., Nugaeva, N., and Hegner, M. 2005. Rapid biosensor for detection of antibiotic-selective growth of Escherichia coli. Appl. Environ. Microbiol. 71:2626-2631.

Gibson, B., Wilson, D. J., Feil, E., and Eyre-Walker, A. 2018. The distribution of bacterial doubling times in the wild. Proc. R. Soc. B. 285: 20180789.

Guilhabert, M. R., and Kirkpatrick, B. C. 2005. Identification of Xylella fastidiosa antivirulence genes: Hemagglutinin adhesins contribute to $X$. fastidiosa biofilm maturation and colonization and attenuate virulence. Mol. Plant-Microbe Interact. 18:856-868.

Hill, B. L., and Purcell, A. H. 1995. Acquisition and retention of Xylella fastidiosa by an efficient vector, Graphocephala atropunctata. Phytopathology 85:209-212.

Hopkins, D. L. 1984. Variability of virulence in grapevine among isolates of the Pierce's disease bacterium. Phytopathology 74:1395-1398.

Killiny, N., and Almeida, R. P. P. 2009. Xylella fastidiosa afimbrial adhesins mediate cell transmission to plants by leafhopper vectors. Appl. Environ. Microbiol. 75:521-528.

Klappenbach, J. A., Dunbar, J. M., and Schmidt, T. M. 2000. rRNA operon copy number reflects ecological strategies of bacteria. Appl. Environ. Microbiol. 66:1328-1333.

Kono, N., Arakawa, K., and Tomita, M. 2011. Comprehensive prediction of chromosome dimer resolution sites in bacterial genomes. BMC Genomics 12:19.

Korem, T., Zeevi, D., Suez, J., Weinberger, A., Avnit-Sagi, T., PompanLotan, M., Matot, E., Jona, G., Harmelin, A., Cohen, N., Sirota-Madi, A., Thaiss, C. A., Pevsner-Fischer, M., Sorek, R., Xavier, R., Elinav, E., and Segal, E. 2015. Growth dynamics of gut microbiota in health and disease inferred from single metagenomic samples. Science 349:1101-1106.

Kurland, C. G. 1991. Codon bias and gene expression. FEBS Lett. 285: 165-169.

Labroussaa, F., Ionescu, M., Zeilinger, A. R., Lindow, S. E., and Almeida, R. P. P. 2017. A chitinase is required for Xylella fastidiosa colonization of its insect and plant hosts. Microbiology 163:502-509.

Langmead, B., and Salzberg, S. L. 2012. Fast gapped-read alignment with Bowtie 2. Nat. Methods 9:357-359.

Li, H., Handsaker, B., Wysoker, A., Fennell, T., Ruan, J., Homer, N., Marth, G., Abecasis, G., Durbin, R., and 1000 Genome Project Data Processing Subgroup. 2009. The sequence alignment/map format and SAMtools. Bioinformatics 25:2078-2079.

Maier, R. M., and Pepper, I. L. 2015. Bacterial growth. Pages 37-56 in: Environmental Microbiology, 3rd ed. I. L. Pepper, C. P. Gerba, and T. J. Gentry, eds. Academic Press, Elsevier, San Diego, CA, U.S.A.

Narang, R., Mohammadi, S., Ashani, M. M., Sadabadi, H., Hejazi, H., Zarifi, M. H., and Sanati-Nezhad, A. 2018. Sensitive, real-time and nonintrusive detection of concentration and growth of pathogenic bacteria using microfluidic-microwave ring resonator biosensor. Sci. Rep. 8: 15807.

Purcell, A. H., and Hopkins, D. L. 1996. Fastidious xylem-limited bacterial plant pathogens. Annu. Rev. Phytopathol. 34:131-151.

R Core Team. 2018. R: A Language and Environment for Statistical Computing. R Foundation for Statistical Computing, Vienna, Austria.

Ramírez, F., Ryan, D. P., Grüning, B., Bhardwaj, V., Kilpert, F., Richter, A. S., Heyne, S., Dündar, F., and Manke, T. 2016. deepTools2: A next generation web server for deep-sequencing data analysis. Nucleic Acids Res. 44:W160-W165.

Rashed, A., Killiny, N., Kwan, J., and Almeida, R. P. P. 2011. Background matching behaviour and pathogen acquisition: Plant site preference does not predict the bacterial acquisition efficiency of vectors. ArthropodPlant Interact. 5:97-106.

Reyes-Lamothe, R., and Sherratt, D. J. 2019. The bacterial cell cycle, chromosome inheritance and cell growth. Nat. Rev. Microbiol. 17: 467-478. 
Rocha, E. P. C. 2008. The organization of the bacterial genome. Annu. Rev. Genet. 42:211-233.

Ruijter, J. M., Ramakers, C., Hoogaars, W. M. H., Karlen, Y., Bakker, O., van den Hoff, M. J. B., and Moorman, A. F. M. 2009. Amplification efficiency: Linking baseline and bias in the analysis of quantitative PCR data. Nucleic Acids Res. 37:e45.

Saponari, M., Boscia, D., Altamura, G., Loconsole, G., Zicca, S., D'Attoma, G., Morelli, M., Palmisano, F., Saponari, A., Tavano, D., Savino, V. N., Dongiovanni, C., and Martelli, G. P. 2017. Isolation and pathogenicity of Xylella fastidiosa associated to the olive quick decline syndrome in southern Italy. Sci. Rep. 7:17723.

Sicard, A., Merfa, M. V., Voeltz, M., Zeilinger, A. R., De La Fuente, L., and Almeida, R. P. P. 2019. Discriminating between viable and membrane-damaged cells of the plant pathogen Xylella fastidiosa. PLoS One 14:e221119.

Smith, H. 1998. What happens to bacterial pathogens in vivo? Trends Microbiol. 6:239-243.

Smolka, M. B., Martins-de-Souza, D., Winck, F. V., Santoro, C. E., Castellari, R. R., Ferrari, F., Brum, I. J., Galembeck, E., Della Coletta Filho, H., Machado, M. A., Marangoni, S., and Novello, J. C. 2003. Proteome analysis of the plant pathogen Xylella fastidiosa reveals major cellular and extracellular proteins and a peculiar codon bias distribution. Proteomics 3:224-237.

Zeilinger, A. R., Turek, D., Cornara, D., Sicard, A., Lindow, S. E., and Almeida, R. P. P. 2018. Bayesian vector transmission model detects conflicting interactions from transgenic disease-resistant grapevines. Ecosphere 9:e02494. 